\title{
Informations
}

Ortho Traumatol (1994) $4: 212$

\section{Janvier 1995}

\section{XVIème Cours de Chirurgie de la Main et du Membre Supérieur de l'Hôpital Bichat (Paris)}

\section{Paris, 26-27 janvier 1995}

Organisé par J.Y. Alnot, C. Oberlin, R. Bleton

Thèmes :

- Pathologies de l'épaule. L'épaule neurologique et paralytique et les traumatismes récents.

- Pathologies traumatiques et non traumatiques du poignet et de la pronosupination

- Actualités chirurgicales

Renseignements et inscriptions: Madame Copin, Secrétaire du Pr J.Y. Alnot, Hôpital Bichat, 46, rue Henri Huchard, F-75877 Paris Cedex 18, Tél. 40257501

\section{GECO 1995}

La 19ème Réunion Elargie du GECO aura lieu à ARC 1800 (Bourg-SaintMaurice) du samedi 14 au vendredi 20 janvier 1995

Sujet des Tables Rondes :

- Instabilités de l'épaule (H. Coudane et J.Cl. Dosch)

- Ostéosynthèses du rachis cervical inférieur (P. Kehr et Ph. Ségal)

Inscriptions: Monique Schweblin, GECO, 24, rue de la Sinne, BP 1232, 68054 Mulhouse Cedex, Tél. 89360532

\section{Avril 1995}

\section{8ème Journées Lyonnaises de Chirurgie du Genou}

\section{Lyon, 6-8 avril 1995}

H. Dejour, J.P. Carret, Ph. Neyret

Centre Hospitalier Lyon-Sud

25 ans de recherches, d'études, d'essais, de résultats... Des lésions ligamentaires, rotuliennes, fémoro-tibiales. La chirurgie prothétique

Renseignements et inscriptions: Nataly Revel, Service du Professeur Dejour, Centre Hospitalier Lyon-Sud, F-69310 Pierre Bénite, Tél. 788614 26, Fax 72392485

\section{4th European Congress on Bone and Joint Infections}

\section{Copenhagen, Denmark, April 27-29, 1995}

Organized by the European Bone and Joint Infection Society

Main topics:
1) Infected total knee arthroplasty
2) Imaging in diagnosis of infection
3) Unproven infections
4) Free papers

Scientific Secretariat and Organization: Dr Carsten Törholm, Department of Orthopaedics, Gentofte Hospital, Niels Andersens Vej 65, DK-2900 Hellerup, Danmark, Tel. 4531651200 , Fax 4331681698 